\title{
Controlling Two-Electron Threshold Dynamics in Double Photoionization of Lithium by Initial-State Preparation
}

\author{
G. Zhu, ${ }^{1}$ M. Schuricke, ${ }^{1}$ J. Steinmann, ${ }^{1}$ J. Albrecht,${ }^{1}$ J. Ullrich, ${ }^{1,2}$ I. Ben-Itzhak,${ }^{3}$ T. J. M. Zouros ${ }^{4}$ J. Colgan, ${ }^{5}$ \\ M. S. Pindzola, ${ }^{6}$ and A. Dorn ${ }^{1}$ \\ ${ }^{1}$ Max-Planck-Institut für Kernphysik, Saupfercheckweg 1, 69117 Heidelberg, Germany \\ ${ }^{2}$ Max-Planck Advanced Study Group at the Center for Free Electron Laser Science, Hamburg, Germany \\ ${ }^{3}$ J. R. Macdonald Laboratory, Department of Physics, Kansas State University, Manhattan, Kansas 66506, USA \\ ${ }^{4}$ Department of Physics, University of Crete, P.O. Box 2208, 71003, Heraklion, Crete, Greece \\ ${ }^{5}$ Theoretical Division, Los Alamos National Laboratory, Los Alamos, New Mexico 87545, USA \\ ${ }^{6}$ Department of Physics, Auburn University, Auburn, Alabama 36849, USA
}

(Received 10 June 2009; published 4 September 2009)

\begin{abstract}
Double photoionization (DPI) and ionization-excitation (IE) of $\operatorname{Li}(2 s)$ and $\operatorname{Li}(2 p)$, state-prepared and aligned in a magneto-optical trap, were explored in a reaction microscope at the free-electron laser in Hamburg (FLASH). From 6 to $12 \mathrm{eV}$ above threshold $(\hbar \omega=85,91 \mathrm{eV})$, total as well as differential DPI cross sections were observed to critically depend on the initial state and, in particular, on the alignment of the $2 p$ orbital with respect to the VUV-light polarization, whereas no effect is seen for IE. The alignment sensitivity is traced back to dynamical electron correlation at threshold.
\end{abstract}

PACS numbers: $32.80 . \mathrm{Fb}$

Understanding the time evolution of correlated manyelectron systems and developing possible schemes to control their dynamics are among the major challenges in physics. Especially intriguing and of fundamental interest is their behavior in extreme situations as, for example, at thresholds. Here, the total kinetic energy available in the final state approaches zero resulting in a subtle balance and, ultimately, in complete correlation between all particles involved, dispensing with any attempt at an independent particle or self-consistent field approximation.

One of the cleanest and most fundamental settings for exploring such phenomena is the double photoionization (DPI) of He. As a result, numerous experimental and theoretical investigations have been reported in the past $[1,2]$ and a profound understanding of He DPI close to threshold has emerged.

For quite some time, the role of the initial state in threshold phenomena has come to the focus of theory. For example, DPI was investigated comparing results for the $\mathrm{He}\left(1 s^{2}{ }^{1} S\right)$ ground state to the $\left(1 s 2 s^{1,3} S\right)$ excited states at high energies [3] as well as close to threshold [4-6]. Significant differences in the total cross sections, their excess energy dependence, and their electron angular distributions were predicted. More recently, interest was stimulated by surprising theoretical results for triple photoionization (TPI) of $\mathrm{Li}$, predicting a nonsymmetric, $T$-shaped final configuration of the emerging electrons at threshold [7] due to specific properties of the Li ground state.

Despite these exciting predictions no DPI experiments have been reported to the best of our knowledge exploring the ground-state dependence, e.g., via investigating (i) excited and aligned initial levels, (ii) nonspherically symmetric states, and (iii) the ejection of electrons from different shells. In the present work on DPI of lithium $\operatorname{Li}\left(1 s^{2} n l\right)$, all of these topics have been pioneered by combining three forefront technologies: A magneto-optical trap (MOT) for Li atoms generating a cold, state-prepared and aligned target, a reaction microscope (REMI [8]) for the high-resolution and large solid angle detection of reaction products and, last but not least, the free-electron laser at Hamburg (FLASH), providing an unprecedented brilliant photon beam at favorable time structure to access vanishing cross sections close to the threshold. Ionizing a $K$-shell electron, mediating the ejection of the valence electron by correlation, we address electrons from different principle quantum levels. Moreover, by means of laser excitation into the $n l=2 p$ state we are able to demonstrate the sensitivity of DPI to the initial target state. Finally, we observe a new phenomenon which so far has not been discussed or predicted in the literature: for $n l=$ $2 p$ the cross section is found to sensitively depend on the spatial alignment of this orbital with respect to the light polarization axis. It is thus demonstrated that a twoelectron transition at threshold can be efficiently controlled through dynamical electron correlation based on a purely geometrical modification of the target initial state without changing its internal energy.

In principle, one might expect that DPI dynamics and transition amplitudes can be controlled by initial-state preparation via two different reaction mechanisms:

(i) Through direct interaction in the "early stage" of ionization within the "reaction zone" where all particles are close together as depicted in Fig. 1: After absorption of a linearly polarized VUV photon the $1 s$ electron is ejected in a dipolelike emission pattern as indicated by the foreground lobes. Subsequently, it might interact with the second electron in the $2 p$ valence shell, eventually leading 


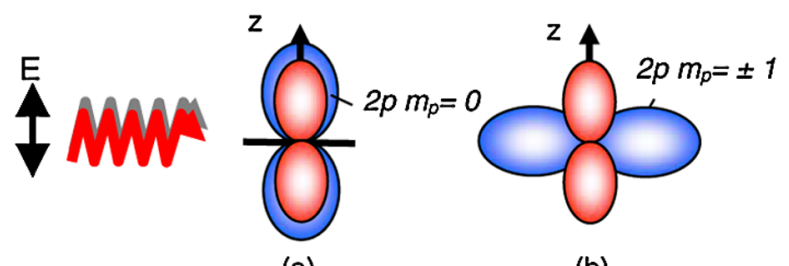

(a)

(b)

FIG. 1 (color online). Intuitive scheme for Li DPI by VUV light linearly polarized along the $z$ axis for different populations (a) $m_{p}=0$ and (b) $m_{p}= \pm 1$ of the magnetic $\left(m_{p}\right)$ sublevels of the excited $2 p$ orbital. For details see text.

to double ionization or IE. As intuitively depicted, DPI might sensitively depend on the alignment of the $2 p$ state. Thus, when aligned along the VUV-polarization a large overlap between the outgoing $1 s$ electron and the $2 p$ density in the $m_{p}=0$ magnetic sublevel [background lobes in Fig. 1(a)] can be expected. The overlap should be much reduced for alignment perpendicular to the VUV polarization [Fig. 1(b)].

(ii) Through long range electron repulsion most important in the vicinity of the threshold where the two ejected electrons are slow. Here, the asymptotic state's quest for stability requires their back-to-back emission. Accordingly, depending on whether this configuration is allowed, "favored" or "unfavored" final-state symmetries have been identified $[9,10]$ with vastly different DPI cross sections, not relying on any short-range interaction.

With our experimental approach we can, thus, choose at will between favored and unfavored final-state symmetries and report compelling alignment sensitivity for DPI, but not for ionization excitation (IE). These results, most pronounced at threshold, point towards the possibility to control two-electron transitions by dynamical correlations near threshold, via reaction mechanism (ii). Even though initial-state manipulation obviously provides a handle to control two-electron continuum cross sections and dynamics, corresponding measurements have not been reported to date, to the best of our knowledge.

Our MOT based Li target contained about $10^{7}$ atoms in a volume of $0.5 \mathrm{~mm}$ diameter at a temperature of roughly $1 \mathrm{mK}$. With a FLASH pulse train containing 30 pulses, each with 20 fs duration and containing up to $10^{13}$ photons at $5 \mathrm{~Hz}$ repetition rate, up to one double ionization event is induced within each pulse for a cross section of $9 \times$ $10^{-21} \mathrm{~cm}^{2}$ at $85 \mathrm{eV}$ photon energy [11]. The ions were extracted by a homogenous electric field and projected onto a time and 2D position sensitive detector. From the position and time of flight information the ion momentum was calculated. For optimal momentum resolution the inhomogeneous magnetic field of the MOT was switched off about $1 \mathrm{~ms}$ before each FLASH pulse train of $150 \mu \mathrm{s}$ duration. Thus, an excellent momentum resolution of 0.05 a.u. was achieved with essentially all target atoms recaptured in the MOT after the pulse train had passed.
Special care was taken in the preparation of the $\operatorname{Li}^{*}\left(2 p^{2} P_{3 / 2}\right)$ excited state by superimposing a dedicated linearly polarized optical pumping laser pulse. The fraction of excited atoms was determined to be $46 \pm 1 \%$ from the decrease of the photoline intensity arising from direct ionization of the valence electron. This process is weaker for the $p$ state by a factor of 30 compared to the $s$ state [12]. The degree of excited state alignment was determined from the observed polarization of the emitted fluorescence radiation. The resulting magnetic sublevel populations in the $l$ basis were $P\left(m_{p}=0\right)=0.68$ and $P\left(m_{p}= \pm 1\right)=0.16$ in the parallel case $(\|)$, where the optical pumping laser polarization is aligned along the VUV polarization. In the perpendicular case $(\perp)$, the populations were $P\left(m_{p}=\right.$ $0)=0.32$ and $P\left(m_{p}= \pm 1\right)=0.34$. While in the present experiment only recoil ion momentum distributions were measured the coincident electron detection, in the future, will allow fully differential studies.

For single ionization in Fig. 2 two-dimensional $\mathrm{Li}^{+}$-momentum distributions projected onto a plane containing the polarization axis $\boldsymbol{E}$ are shown. Since the momenta of the absorbed photons are negligible the ion balances the momentum of the ejected electron. Thus, here the ion momentum spectra are equivalent to the electron momentum distributions. For ionization from the Li ground state [Fig. 2(a)] three circular shells are visible with the characteristic dipole double-lobe angular distribution aligned along $\boldsymbol{E}$. Going from large to small radii, the shells correspond to ionization of the $2 s$, one $1 s$ electron and $1 s$ ionization with simultaneous excitation (IE) of the valence electron $2 s \rightarrow n l$, respectively. In Fig. 2(b) the spectrum for the pure $\operatorname{Li}(2 p)$ initial state shows significant changes in the line intensities. As mentioned above ejection of the valence electron is strongly reduced. Furthermore, the cross section for IE increases from $23 \%$ of the pure $1 s$-ionization cross section for $\mathrm{Li}(2 s)$ to $70 \%$ for Li $(2 p)$. Cubaynes et al. [13] observed this behavior even

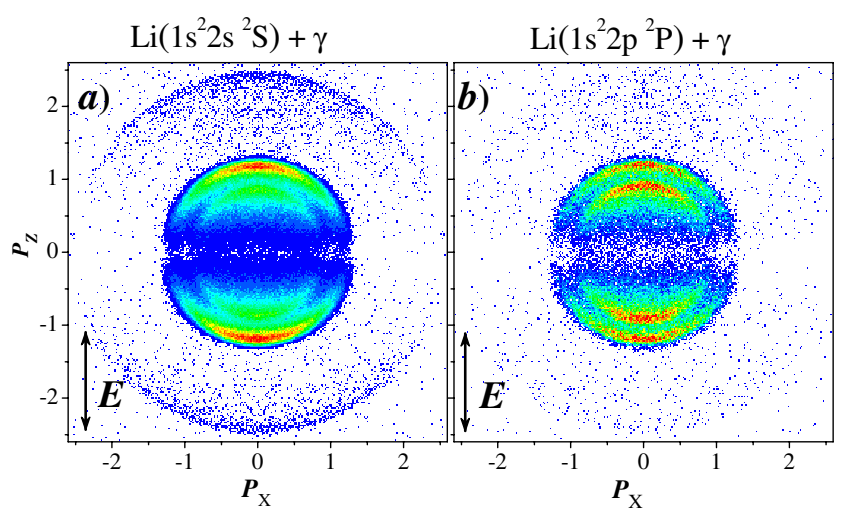

FIG. 2 (color online). Two-dimensional recoil ion momentum distributions for single ionization by $85 \mathrm{eV}$ FEL light of (a) $\mathrm{Li}(2 s)$ and (b) laser excited $\mathrm{Li}(2 p)$. Here the laser and VUV-light polarizations are parallel as indicated by the arrows. 
more pronounced for higher excited initial $n l$ levels. Since the initial-state alignment of their target was not determined, no conclusive result on the alignment dependence was obtained. While in the present measurement the different residual ionic states for IE are not resolved the total IE cross section does not show any dependence on the alignment of the initial valence orbital within the statistical accuracy of $1 \%$. On one hand this is expected for the shakeup transitions $2 p \rightarrow n p(n>2)$ which constitute roughly $80 \%$ of the IE intensity [13]. On the other hand this indicates that also the conjugate shakeup transitions $2 p \rightarrow n s, n d$ which involve an explicit interaction of both active electrons in the final state do not show an alignment dependence signifying that mechanism (i) discussed above is of minor relevance.

Going to double ionization we first demonstrate how the process is influenced by the symmetry of the initial state and how initial-state preparation can modify the electron pair emission geometry. We consider the following transitions for the Li ground and excited states,

$$
\begin{aligned}
\gamma+\operatorname{Li}\left(1 s^{2} 2 s^{2} S^{e}\right) & \rightarrow \mathrm{Li}^{2+}\left(1 s^{2} S\right)+2 e^{-1,3} P^{o} \\
\gamma+\operatorname{Li}\left(1 s^{2} 2 p^{2} P^{o}\right) & \rightarrow \mathrm{Li}^{2+}\left(1 s^{2} S\right)+2 e^{-1,3} S^{e},{ }^{1,3} D^{e}
\end{aligned}
$$

respectively, for which the obtained recoil ion momentum distributions are shown in Fig. 3. Here, both the VUV radiation and the optical pumping laser radiation are polarized parallel to one another such that only $\Delta M=$ 0 transitions can be induced with respect to this quantiza-

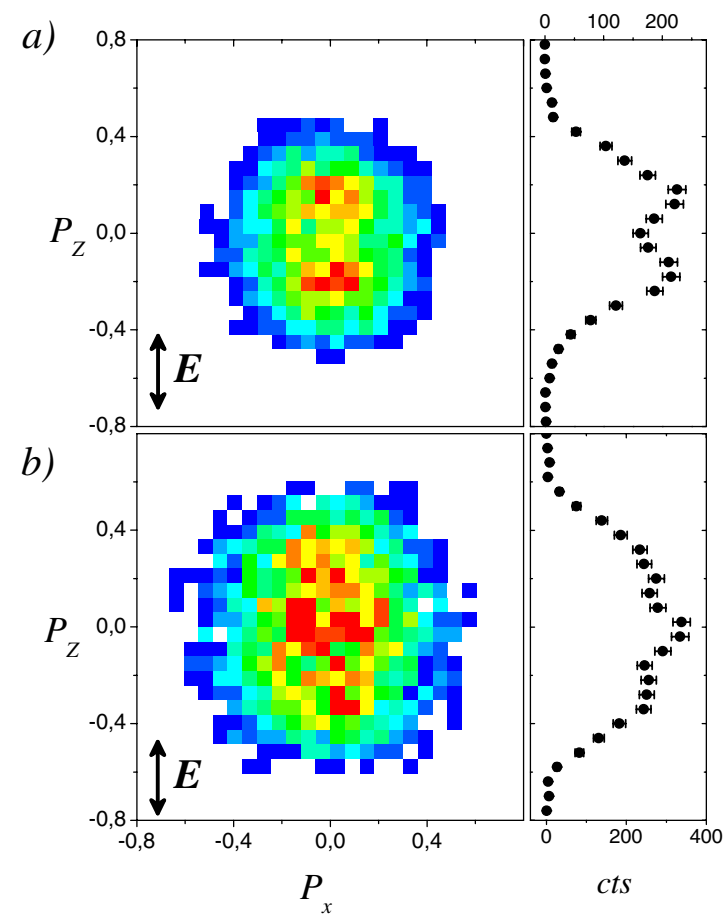

FIG. 3 (color online). Same as for Fig. 2, but for double ionization. Projections of the momentum distributions onto the vertical axis are shown in the diagrams to the right. tion axis. From the accessible final states the singlet states dominate the total DPI cross section as it was demonstrated previously by calculations for DPI of excited helium $\mathrm{He}^{*}\left(1 s 2 s^{1,3} S^{e}\right)[4-6]$ which obtained a cross section ratio $\sigma\left({ }^{1} P\right) / \sigma\left({ }^{3} P\right) \approx 10$ in the region 5 to $10 \mathrm{eV}$ above threshold. For clarity, we consider only the singlet states in the following analysis.

Figure 3(a) shows a double-lobe pattern with a minimum at $\left|\boldsymbol{P}_{\text {rec }}\right|=0$. As for DPI of helium this follows from the ${ }^{1} P^{o}$ symmetry of the outgoing electron pair's partial wave which has a node at the spatially symmetric Wannier configuration. Accordingly, ${ }^{1} P^{o}$ has an unfavored symmetry with vanishing cross section for back-to-back electron emission with equal energies corresponding to zero recoil ion momentum. In contrast, the outgoing ${ }^{1} S^{e}$ and ${ }^{1} D^{e}$ waves $(M=0)$ reached from the excited initial state correspond to favored symmetries allowing the Wannier configuration. Furthermore, these states are the only ones up to $L=3$ for which no selection rule, i.e., no emission geometry with vanishing cross section was identified in the analysis of Maulbetsch and Briggs [14]. As a result, in Fig. 3(b) the minimum at $\left|\boldsymbol{P}_{\text {rec }}\right|=0$ is filled up and converted into a cross section maximum. So far, such favored symmetries could only be reached for DPI of the spherically symmetric argon atom [15].

We next rotate the alignment of the $2 p$ orbital with respect to the VUV polarization from parallel $\left(2 p_{\|}\right)$to perpendicular $\left(2 p_{\perp}\right)$. As a result the DPI rate decreases significantly for small ion momenta below 0.6 a.u., as can be seen in Fig. 4(a) where the cross section as a function of the recoil ion momentum is plotted. Interestingly this alignment sensitivity decreases strongly as the photon excess energy increases from 6 to $12 \mathrm{eV}\left(E_{\gamma}=91 \mathrm{eV}\right)$ as shown in Fig. 4(b). Thus, this effect is not enforced by symmetry, but rather by a subtle dynamical correlation which is more effective closer to the double ionization threshold. These observations support the above mentioned mechanism (ii), indicating long range correlation at threshold to be at work: DPI at vanishing excess energy requires that all available energy is used to overcome the ionic potential well. For perpendicular alignment of the $2 p$ orbital the initial state contains considerable momentum transverse to the preferred emission angle of the photoionized innershell electron along the VUV-polarization axis. As a result the ejected electron pair has a finite sum momentum or center of mass motion which does not fulfill the threshold condition of a symmetric back-to back emission ([16], and references therein). This intuitive picture is represented by the selection rule "B1" compiled by Maulbetsch and Briggs [14] stating that two-electron symmetries with $M \neq 0$ do not contribute to the cross section for emission of both electrons along the quantization axis, i.e., the VUV-polarization axis. While for the initial $2 p$ $(m=0)$ state for the ejected electron pair two partial wave symmetries $\varepsilon S\left(M_{S}=0\right)$ and $\varepsilon D\left(M_{D}=0\right)$ can be 


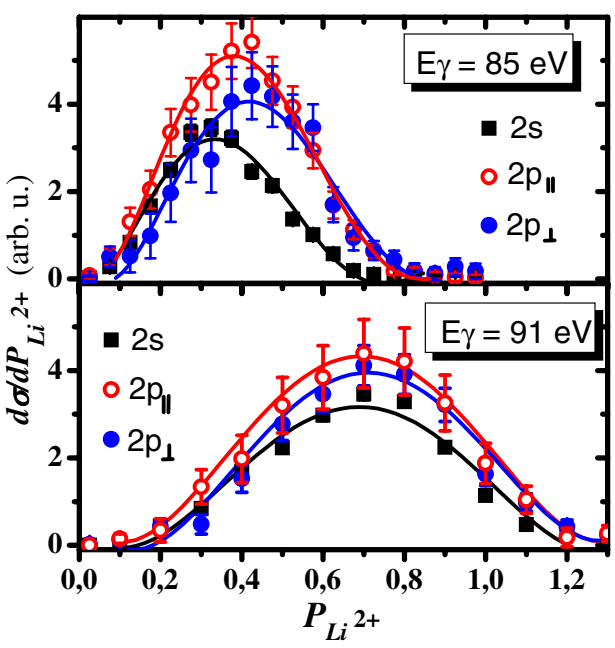

FIG. 4 (color online). Double ionization recoil ion momentum distributions as a function of total $\mathrm{Li}^{2+}$ momentum by (a) $85 \mathrm{eV}$ and (b) $91 \mathrm{eV}$ photons for the three different initial states indicated. The lines are to guide the eye.

reached, for perpendicular alignment with $2 p m= \pm 1$, the final $S$ partial wave is not allowed but two $D$ waves with $M= \pm 1$ contribute. Thus, different transition amplitudes are involved in both cases enabling an alignment dependence of the cross section which, however, is reduced for increasing photon energy and completely absent if one of the two electrons stays bound.

These arguments are supported by time-dependent close-coupling calculations (TDCC) of the fully differential cross section. The TDCC method [17] is used to compute the DPI of Li after "freezing" one of the $1 \mathrm{~s}$ electrons. Singlet and triplet contributions from the coupling of the outgoing electron pair are included after appropriate recoupling. At $85 \mathrm{eV}$ photon energy the calculation yields considerably higher cross sections for the $\varepsilon S\left(M_{S}=0\right)$ and the $\varepsilon D\left(M_{D}=0\right)$ partial waves compared to the $\varepsilon D\left(M_{D}= \pm 1\right)$ partial waves. For increasing excess energy this difference diminishes. In Table I the change of the total double ionization cross section for the differently aligned excited states relative to the groundstate cross section is shown for the experiment and the TDCC theory. The values agree within $12 \%$ except for the $p_{\perp}$ case at $91 \mathrm{eV}$ photon energy where theory is $30 \%$ below the experiment.

While in the present, pioneering experiment the cross section was modified by up to $34 \%$ for the different pumping laser polarizations, an even higher contrast is expected closer to threshold and for an increased population of the $2 p\left(m_{p}= \pm 1\right)$ initial substates, reachable via optical pumping with circularly polarized light. Then, DPI is either enabled or essentially suppressed via controlling dynamical electron correlation through a purely geometrical modification of the target initial state without changing its internal energy.
TABLE I. Experimental and theoretical total DPI cross sections for the differently aligned excited state normalized to the ground-state DPI cross section.

\begin{tabular}{cccc}
\hline \hline Photon energy & Initial state & Experiment & TDCC \\
\hline $85 \mathrm{eV}$ & $2 p_{\|}$ & $1.74(5)$ & 1.52 \\
& $2 p_{\perp}$ & $1.30(4)$ & 1.17 \\
$91 \mathrm{eV}$ & $2 p_{\|}$ & $1.34(5)$ & 1.22 \\
& $2 p_{\perp}$ & $1.18(4)$ & 0.90 \\
\hline \hline
\end{tabular}

In conclusion, initial-state preparation of the target is demonstrated to provide an important handle for controlling two-electron continuum dynamics at threshold leading to significantly different DPI cross sections. While this was predicted theoretically for excited initial states the alignment dependence is a completely new phenomenon. It is traced back to the long range correlation between the Coulomb interacting particles which dominates the dynamics close to threshold.

We are greatly indebted to R. Treusch, S. Düsterer, and all other members of the scientific and technical team at FLASH. The Los Alamos National Laboratory is operated by Los Alamos National Security, LLC for the National Nuclear Security Administration of the U.S. Department of Energy under Contract No. DE-AC5206NA25396. This work was supported in part by the Chemical Sciences, Geosciences, and Biosciences Division, Office of Basic Energy Sciences, Office of Science, U.S. Department of Energy.

[1] L. Avaldi and A. Huetz, J. Phys. B 38, S861 (2005).

[2] P. Bolognesi, G. King, and L. Avaldi, Radiat. Phys. Chem. 70, 207 (2004).

[3] Z.J. Teng and R. Shakeshaft, Phys. Rev. A 49, 3597 (1994).

[4] H. W. van der Hart, K. W. Meyer, and C. H. Greene, Phys. Rev. A 57, 3641 (1998).

[5] A. S. Kheifets et al., Phys. Rev. A 62, 052724 (2000).

[6] J. Colgan and M. S. Pindzola, Phys. Rev. A 67, 012711 (2003).

[7] A. Emmanouilidou, P. Wang, and J. M. Rost, Phys. Rev. Lett. 100, 063002 (2008).

[8] J. Ullrich et al., Rep. Prog. Phys. 66, 1463 (2003).

[9] A. D. Stauffer, Phys. Lett. A 91, 114 (1982).

[10] C. H. Greene and A. R. P. Rau, Phys. Rev. Lett. 48, 533 (1982).

[11] R. Wehlitz, J. B. Bluett, and S. B. Whitfield, Phys. Rev. A 66, 012701 (2002).

[12] B. Najjari (private communication).

[13] D. Cubaynes et al., Phys. Rev. Lett. 99, 213004 (2007).

[14] F. Maulbetsch and J. S. Briggs, J. Phys. B 28, 551 (1995).

[15] J. Mazeau, P. Lablanquie, P. Selles, L. Malegat, and A. Huetz, J. Phys. B 30, L293 (1997).

[16] J. M. Rost and J. S. Briggs, J. Phys. B 24, L393 (1991).

[17] M. S. Pindzola et al., J. Phys. B 40, R39 (2007). 